Vivre, penser, écrire en exil

\title{
Les espaces de l'intellectuel en exil : trajectoires et réseaux immigrés dans le Paris d'après-guerre (1945-1960)
}

Exiled Intellectuals Spaces: Immigrant Paths and Networks in Afterwar Paris (1945-1960)

Los espacios del intelectual en exilio: trayectorias y redes de inmigrantes en el París de posguerra (1945-1960)

\section{Jérémy Guedj}

\section{OpenEdition}

\section{Journals}

Édition électronique

URL : https://journals.openedition.org/remi/8566

DOI : 10.4000/remi.8566

ISSN : $1777-5418$

Éditeur

Université de Poitiers

Édition imprimée

Date de publication : 1 mars 2017

Pagination : 27-47

ISBN : 979-10-90426-30-6

ISSN : 0765-0752

\section{Référence électronique}

Jérémy Guedj, « Les espaces de l'intellectuel en exil : trajectoires et réseaux immigrés dans le Paris d'après-guerre (1945-1960) », Revue européenne des migrations internationales [En ligne], vol. 33 - n¹ I 2017, mis en ligne le 01 mars 2019, consulté le 14 avril 2022. URL : http://journals.openedition.org/ remi/8566; DOI : https://doi.org/10.4000/remi.8566 


\section{Les espaces de l'intellectuel en exil : trajectoires et réseaux immigrés dans le Paris d'après-guerre (1945-1960)}

\section{Jérémy Guedj'}

« Et Paris ne fut plus jamais le même. C'était pourtant toujours Paris, et s'il changeait, vous changiez en même temps que lui " (Ernest Hemingway).

"Paris est une ville dont on pourrait parler au pluriel, comme les Grecs parlaient d'Athènes, car il y a bien des Paris, et celui de l'étranger n'a que des rapports de surface avec le Paris des Parisiens ", affirmait Julien Green depuis les États-Unis, tandis que la guerre courait vers son terme (Green, 1998 : 1118) ${ }^{2}$. Élégamment formulée, cette remarque n'avait cependant rien de neuf. Au XIXe siècle déjà, Tocqueville l'avait clairement mise en mots : "II existe au sein de Paris une masse flottante composée d'écrivains plus ou moins désordonnés dans leurs habitudes, de femmes auteurs ou d'une réputation compromise, $d^{\prime}$ artistes en tout genre, de journalistes, de caractères singuliers et entreprenants que les chances de l'ambition ou le plaisir attirent de tous les coins de la France, multitude bigarrée qui outre toutes les qualités et tous les défauts du caractère national et qui ne représente qu'elle-même. Non seulement cette société n'offre point l'image exacte de la France, mais elle ne donne pas même une idée vraie de Paris. Or, c'est au sein de cette société qu'un étranger, quels que soient son esprit et sa distinction, est presque toujours et presque exclusivement entraîné " $^{3}$. À lire ces témoignages, on comprend ce que le "Paris des étrangers " (Kaspi et Marès, 1989 ; Marès et Milza, 1994), plus particulièrement les intellectuels parmi eux, avait d'unique, et d'artificiel. II existait toutefois autant de géographies de l'exil que d'expériences migratoires. Paris, métonymie de l'exil des gens de lettres, car c'était là que convergeaient le plus souvent les trajectoires d'intellectuels venus d'ailleurs, n'offrait pas le même visage, ni la même vie, à tous ceux qui en faisaient leur ville d'élection. Les années d'aprèsguerre, époque de renouvellement des milieux de l'esprit, l'illustraient avec toujours autant d'acuité.

\footnotetext{
1 Docteur en histoire contemporaine, Chercheur rattaché au Centre de la Méditerranée moderne et contemporaine (CMMC), Enseignant à I'Université Nice-Sophia-Antipolis et à Sciences-Po Paris (campus de Menton), 98 boulevard Édouard Herriot, BP 3209, 06204 Nice cedex; jeremy.guedj@unice.fr

2 Ce texte fut lu à la radio tandis que son auteur travaillait pour l'Office of War Information.

3 Cité par Slama (1998: 124) et extrait de Tocqueville Alexis de (1954 [1837]) Note sur un livre d'Henry Bluwer, in Alexis de Tocqueville, Fuvres complètes, t. IV, Correspondance anglaise, Paris, Gallimard, p. 323.
} 
Distinguer le Paris des intellectuels étrangers de celui des autres migrants sans adjectif ou que, par abus de langage, on qualifie trop rapidement d'économiques et politiques - risque, derrière le voile des fausses évidences, de pervertir la compréhension. Et de trop insister sur la première appartenance, comme si elle gommait les autres faisceaux d'identité. Or, l'entrée dans un milieu social et culturel, celui où l'activité principale résidait dans les choses de l'esprit, n'avait a priori rien d'évident pour les étrangers, quand bien même ils se consacraient pleinement à cela. Qui parle d'intellectuel étranger est sommé de réexplorer le sens de ces termes à l'aune de l'interaction que l'un exerce sur l'autre. Émettons I'hypothèse selon laquelle, en exil, les intellectuels s'ancraient dans des espaces et réseaux, intellectuels et étrangers, qui ne se rencontraient que partiellement. Une reconfiguration des frontières identitaires marquait leur expérience, entre appartenance culturelle et origine nationale (Guedj, 2015a). De sorte que l'intellectuel étranger devient dès lors un double archétype, car deux fois " décentré " (Laronde, 1999) : entre le milieu des lettres et de la pensée - lieu du décalage par excellence ${ }^{4}$ - et les contingences d'un espace ou d'un temps ; mais aussi entre la terre - ici la ville - d'accueil et le legs des origines. Aussi vivait-il à la fois deux " conditions", pour reprendre un terme auquel les sciences sociales donnent une nouvelle jeunesse (Ndiaye, 2008). Tension, voire contradiction, au cœur de la situation vécue par ces intellectuels étrangers qui se voient dans " l'obligation de jouer en partie double, d'être ici et ailleurs, d'occuper deux lieux à la fois, ce qui contraint à rester dans l'entre-deux " (Dollé, 2001: 135).

Histoire singulière et plurielle que celle de ces intellectuels exilés, qui implique une analyse en termes de parcours individuels et collectifs, sous I'angle de la nationalité notamment. La production, qui compose un vaste corpus, n'en est qu'une des voies d'entrées, à compléter par des expériences vécues, dont les écrits ne conservent pas toujours la trace et qui se dérobent parfois à l'analyse.

\section{Les Paris de l'exil intellectuel ou l'infinie déclinaison d'une cité-carrefour}

"Méridien de Greenwich littéraire ", "ville-littérature ", selon Pascale Casanova (1999: 127 et 41), Paris prête le flanc à tous les topoï, validés à chaque génération, avec quelque inflexion parfois, par les étrangers qui maniaient la plume. Cela tenait parfois plus du mythe que de la réalité. Relisons sur ce point Roger Caillois : à l'œuvre, selon lui, " une représentation de la grande ville, assez puissante sur les imaginations pour que jamais en pratique ne soit posée la question de son exactitude, créée de toutes pièces par le livre, assez répandue néanmoins pour faire maintenant partie de l'atmosphère mentale collective et posséder par suite une certaine force de contrainte "(Caillois, 1938 : 153). Les intellectuels allogènes qui arrivaient à Paris apparaissaient donc pétris de cette image, qu'ils tenaient d'une culture livresque confrontée, dans un second temps, à la réalité. II convient donc de ne pas sacrifier à la causalité unique : Paris attirait, conservait son lustre, mais il était diverses manières de rejoindre et de vivre cette ville, où tout ne se résumait pas à une admiration lyrique. Comme le

4 "Nous pensons toujours ailleurs ", écrivait Montaigne dans " De la diversion ", Essais (Livre III, chap. IV). Sur ce point, voir le beau livre de Lapierre (2004).

5 Cité par Delbart, $2005: 52$. 
souligne Ralph Schor (1989 : 33) : "Derrière [la] lumière s'étendait une ombre, plus ou moins épaisse. [...] Pourtant, même si la réputation de Paris paraissait surfaite, les étrangers continuaient d'y affluer. C'était d'abord parce que l'éclat de la grande métropole brillait si fort qu'il cachait certaines scories ; Paris vivait sur sa lancée, sur les acquis du passé, sur un prestige ancien ".

Cette longévité, prompte à alléger le poids de la rupture, gardait toute sa force après la Seconde Guerre mondiale, dans la capitale d'un pays affaibli, à la réputation écornée. Les stéréotypes ne s'encombraient pas des convulsions historiques. Un dialogue mérite d'être reproduit in extenso, tant il rassemble à lui seul nombre de paramètres à l'œuvre; il s'agit d'un extrait de La Chambre de Giovanni, roman, aux accents autobiographiques, de l'écrivain américain installé en France, James Baldwin. L'échange de vues, animé, opposait le narrateur au héros éponyme, qui avait ouvert la discussion :

" - “Vous êtes américain? demanda-t-il enfin.

- Oui. De New York.

- Ah! On dit que New York est une très belle ville. Est-ce que c'est plus beau que Paris?

- Oh, non. Il n'y a pas de ville au monde qui soit plus belle que Paris.

- On dirait que ça vous met en colère qu'on puisse même le suggérer." Giovanni souriait

largement. "Pardonnez-moi, je ne savais pas que je disais une hérésie". Puis, plus sobre, comme pour me tranquilliser, il ajouta: "Vous devez beaucoup aimer Paris.

- J'aime New York aussi, dis-je sur la défensive, et embarrassé de l'entendre de ma voix, mais New York est beau d'une manière très différente."

Il fronça les sourcils. "Comment ça?

- C'est une ville qu'on ne peut absolument pas imaginer avant de l'avoir vue. C'est très haut, neuf et éclectique, très enivrant. C'est difficile à écrire. C'est très... xx siècle.

- Vous trouvez que Paris n'est pas de ce siècle ?" demanda-t-il avec un sourire.

À cause de ce sourire, je me sentis un peu bête.

- “C'est que Paris est vieux, il a plusieurs siècles. À Paris, on sent le temps qui a passé.

On ne ressent pas ça à New York" " (extrait de Baldwin, $1998: 45)^{6}$.

On retrouve ici l'exaltation de la France, son passé qui se perd dans la légende - vérité si indiscutable qu'il paraissait hors de propos d'en douter - et la place de choix occupée par Paris. Ce rapport, discordant dans le cas de la Ville-lumière, entre le temps et l'espace, ne manque pas de frapper ${ }^{7}$. Certes, les allusions, concernant plus la France entière que Paris nommément, étaient nombreuses, qui soulignaient les affres de l'époque et leurs conséquences sur la terre d'asile ; Malaparte (1967:15) parlait du " Paris fatigué de 1947, bien plus cher à mon cœur que le Paris de $1933 n^{8}$. Mêlant l'image de la capitale et celle du pays, inextricablement liées, Antoine Marès explique, à propos des exilés d'Europe centrale : "Après 1948, la destination "naturelle" des exilés tchèques

6 La comparaison entre Paris et New York semble renvoyer à une forme de concurrence des topoï, la seconde ville jouant aussi le rôle de " mythe littéraire ". Voir Pinçonnat (2001).

7 Sans doute s'agissait-il d'une forme d'infléchissement par rapport aux images qui prévalaient avant-guerre, où Paris se voyait associer également une forme de modernité artistique. "Paris était là où était le XXe siècle ", avait écrit une autre Américaine, Stein (1941 : 23). Sur ce point, voir Schor (1985 : 200).

8 Les Américains partageaient tout particulièrement cet attrait, eux qui avaient quitté un pays neuf pour le "Vieux Continent ". Voir Baldwin (2000) et les chroniques de Flanner (1985), correspondante du The New Yorker, sous le pseudonyme de Genêt. 
n'est plus Paris. Les accords de Munich, qui continuent à être considérés par beaucoup comme un abandon impardonnable, et la défaite de la France en juin 1940 ont terni l'image de la grande puissance qui avait contribué à la naissance de l'État tchécoslovaque " (1994: 150). Tournant qui se situe au même moment pour les Américains : "Au fil des années, le crédit libéral de la France diminuera ou augmentera selon les circonstances ; néanmoins, et globalement, la fascination restera puissante et Paris brillera d'un éclat prestigieux tout au long du XIXe siècle. Ce même rayonnement perdurera jusque dans les années 1950, malgré des déconvenues plus ou moins graves" (Fabre, 1985 : 24).

Nonobstant cet arrière-plan, le bouleversement ne semblait pas clairement perceptible. Rapporté à l'ensemble des nationalités présentes, il se produisait par petites touches progressives, mais en aucun cas brutales. Pour peu que I'on prête, ce qui pose question, un "principe de sincérité " (Sapiro, 2004) à la production rassemblée, on se rend compte de l'étonnante permanence, qui traverse les nationalités, du Paris libéral et artistique.

Un motif récurrent était celui de la culture artistique et intellectuelle qu'incarnait Paris, capitale d'un pays aux mœurs délicates, raffinées en toute circonstance ; cela avait frappé les étrangers de longue date (Schor, 2013 : chap. II). Arrivés en France, ceux parmi ces derniers qui avaient des prétentions littéraires ou artistiques s'inscrivaient ainsi dans une illustre généalogie. Le communiste allemand Georges Glaser, ouvrier qui tenta de se frayer un chemin dans le milieu littéraire, sans succès, rappelait : "Dans ma petite patrie, le nom de "Paris" sonnait comme un mot de légende. Plus tard, mes lectures et mes expériences ne le dépouillèrent pas de cet éclat. C'était la ville d'Henri Heine, la ville de JeanChristophe, la ville d'Hugo, de Balzac, de Zola, la ville de Marat, Robespierre, Danton, la ville des éternelles barricades et de la Commune, la ville de l'amour, de la lumière, de l'air léger, du rire et du plaisir. Et bien que j'eusse marché plusieurs jours en direction de la ville, je sentis que c'était seulement à la vue du merveilleux poteau indicateur que j'avais commencé à croire à Paris, comme à une ville habitée, et réellement accessible " (Glaser, 1951 :157). Vincent Carter, revenu à Paris en 1954 après un bref passage en tant que Gl après la guerre, demandait : "Paris n'est-il pas le centre des arts ? Tous les grands écrivains n'y avaient-ils pas séjourné ? Heine, Rilke, Hemingway ? " ${ }^{9}$. À chaque exemple son contre-exemple ? Parlons plutôt de nuances : si I'arrivée à Paris prenait parfois de troublants airs de pèlerinage, Crystel Pinçonnat souligne, reprenant Gérard Genette (1982 : 290), un certain " refus d'hériter " que manifestait plus d'un écrivain afro-américain de ce que l'on a appelé la "Seconde Génération perdue "; cela afin de se distinguer de leurs aînés blancs et de se rapprocher de la culture noire et de la " négritude ", très en vogue dans le Paris de l'aprèsguerre, nous y reviendrons (Pinçonnat, 2000 : 1 068). Les lignes bougeaient, mais l'essentiel demeurait. Le décor de la capitale, presque onirique sous la plume des écrivains, constituait l'écrin privilégié de la création. Julien Green (1975 : 707), ayant gagné les États-Unis pendant la guerre, écrivait dans son journal (27 janvier 1943) : " Jamais je ne m'accoutumerai à l'absence de Paris " ; " II faut que j'aie Paris autour de moi pour écrire ", ajoutait-il le 4 septembre 1944 (Green, 1975 : 804).

9 Cité par Fabre (1985: 152) et extrait de Carter Vincent (1973), The Bern Book, New York, John Day, p. 7. 
La liberté et le climat privé d'entraves se retrouvaient sur le plan politique et régissaient les relations sociales ou ethniques. Qu'ils en fussent dépourvus ou non dans leur pays d'origine, la liberté, l'ouverture et l'absence de contraintes ressenties, qu'il faudrait nuancer dans les faits (Schor, 1989 ; Guedj, 2015b), faisaient l'objet d'éloges appuyés de la part des étrangers. L'atmosphère semblait propice à l'affaissement des barrières entre individus d'origines diverses. Richard Wright, écrivain noir américain arrivé en France en 1946 pour échapper aux poursuites américaines en raison de son communisme, se surprit d'entendre un Gl, également afro-américain, aux bras d'une jeune Française blanche, lui dire qu'il comptait rentrer avec elle à Saint-Louis, en Louisiane, où le déchaînement raciste nourrissait les chroniques quotidiennes : " Je le regardai avec stupeur. Avait-il oublié quelle sorte de ville était Saint-Louis ? Ne savait-il pas que ce serait la mort pour lui s'il revenait accompagné d'une femme blanche ? [...] Et je compris tout. Ce gentil garçon, ce brave noir américain avait oublié... Vivant à Paris depuis deux ans, il avait appris à connaître et à aimer la liberté. Les rigides lois raciales avaient perdu, pour lui, leur réalité ${ }^{10}$. Quelques années plus tard, le même Richard Wright ratifiait, dans la revue The Crisis, un constat semblable, rappelant que le Noir américain en France " vaque à sa routine quotidienne sans I'angoisse de devoir affronter les assauts de l'arbitraire racial $n^{11}$, mais notait une certaine dégradation, liée à une forme de repli fermant les yeux sur l'importation du mal américain, la ségrégation, à I'heure du raidissement des blocs ${ }^{12}$. Allégorie de la liberté, Paris devenait un être à part entière, que I'on pouvait distinguer de ses habitants. Le Hongrois François Fejtö (1986) en retraçait le sentiment : "Dire que la France m'accueillit à bras ouverts serait exagéré. [...] Paradoxalement, l'étranger se sent tout de suite chez lui à Paris et met longtemps à s'apercevoir que les Parisiens ne l'acceptent pas ". Poussons plus loin I'hypothèse, pour soutenir que ce rapport direct à la ville, négligeant la population, expliquait tout autant le lien charnel qui unissait cet espace à ses étrangers et le sentiment d'ouverture à l'autre ressenti par ces derniers. Les pages aux accents lyriques de la Russe Nina Gourfinkel, éloignée de sa ville, pour le délit d'être née juive, pendant les années noires, semblent valider une telle image : "Cinquante-deux mois d'exil : tout ce qui n'est pas Paris est exil ", écrivait-elle avant d'ajouter : "C'est quand sa hantise semble m'avoir relâchée que je me sens le plus près de Paris. La pensée de Paris - émanation de la France, qui m'est apparue confusément durant mes quinze premières années françaises et qu'avait refoulée le Paris mondial, international, complexe, de mes curiosités et de mon travail, se concrétisait : ville éminemment française, nourrie des forces vives du pays, de la sève qui sans cesse remonte vers lui comme le sang vers le cœur. [...] Et pourtant, cette force intérieure qui, ici, semble se suffire à elle-même ne saurait à elle seule produire Paris. Elle donne les racines et la tige, mais la fleur devient fruit grâce au pollen du monde. De ce pays replié

10 Cité par Fabre (1985: 150) et extrait de Wright Richard (1946) À Paris, les GI noirs ont appris à connaître et à aimer la liberté, Samedi Soir, 25 mai, p. 2.

11 Wright Richard (1951) American Negroes in France, The Crisis, juin-juillet 1951, p. 381. La revue The Crisis, fondée en 1910 par William Dubois, en lien avec la National Association for the Advancement of Colored People (NAACP), fut par la suite marquée par les réactions d'indignation françaises à I'affaire Emmett Till, assassiné en 1955 dans le Mississippi (L'affaire Till in the French Press, The Crisis, novembre 1955, pp. 596-602).

12 L'écrivain Richard Wright se plaint de discriminations raciales à I'hôpital de Neuilly, Combat, 8 juin 1951 ; Logique du Pacte atlantique : à I'hôpital américain de Neuilly, on cultive le virus raciste, L'Observateur, 15-20 juin 1951. 
sur ses richesses, Paris est la porte ouverte de l'univers, la seule par laquelle il soit permis à l'étranger d'entrer. Nous autres, nous pouvons être naturalisés Parisiens, non Français " (Gourfinkel, 1953 : 319-320).

Aux nombreux éloges, répondaient des réserves, moins fournies et sans doute représentatives d'un moindre échantillon d'intellectuels, mais qui n'en venaient pas moins pondérer et nuancer le tableau monolithique que l'on serait enclin à trop vite brosser. La déception guettait parfois, peut-être en raison d'attentes trop élevées. Le Péruvien Sebastián Salazar Bondy ne mâchait pas ses mots et, entamant un cycle d'études dans la capitale française en 1956, écrivait : "J'étais frappé par l'aliénation des étudiants et artistes qui partaient vivre à Paris. Paris, selon Hemingway, était une fête, mais je n'y trouvais aucune semblable fête ${ }^{\prime 13}$. La perception de Paris - et, partant, la façon d'y vivre et de $\mathrm{s}^{\prime}$ approprier, ou non, cet espace - dépendait grandement du profil et de l'origine des intéressés. Observation particulièrement flagrante dans le cas des migrants, étudiants et intellectuels, issus des colonies. L'heure était alors aux guerres d'indépendance et établissait une relation d'attraction mâtinée de répulsion. Dans Le Sommeil du juste, Mouloud Mammeri (1957) croque un jeune étudiant dans le Paris d'après-guerre, émerveillé par le musée à ciel ouvert qu'il foule chaque jour, lequel voisinait avec les sordides logements destinés à accueillir les immigrés algériens venus travailler à la reconstruction de la France, dont I'invisibilité n'avait d'égale que la misère ${ }^{14}$. Quant à Khaled, l'écrivain héros du roman de l'Algérien Malek Haddad, Le Quai aux fleurs ne répond plus, il ne pouvait s'empêcher de penser au drame qui frappait son pays, tandis que les voitures de police jonchaient la capitale et rappelaient en permanence l'état d'urgence. Rester à Paris, c'était refuser la réalité ; et refuser la réalité, c'était trahir les frères demeurés de l'autre côté de la Méditerranée ; "Paris, c'est une mauvaise habitude à prendre ", clamait-il (Haddad, 1961 : 27). La conjoncture, I'Histoire ne dictaient pas tout. Le sentiment d'altérité faisait aussi son œuvre. Ainsi marquait-il Mohammed Dib :

"Soirs tendres de Paris, que vous m'êtes amers.

Pour l'exilé, Paris obscur, c'est un enfer :

Quand le ciel gris et rose au-dessus de la Seine Se repose en tremblant, tout son cœur crie et saigne.

Quel étranger ici ne se sent pas chez lui? Mais ça vous prend ainsi dès que tombe la nuit, Sa place on ne l'a pas dans cette ville immense, Croit-on, c'est le mauvais rêve qui recommence " (extrait de Dib, 1958) ${ }^{15}$.

Ambivalence que l'on retrouvera, de façon plus marquée encore, parmi les

13 Cité par Flores (1992: 793) et repris dans Weiss (2003: 76).

14 Voir l'analyse de Arnaud (1992: 198).

$15 \mathrm{La}$ situation coloniale n'était pas seule en cause dans l'évocation de Paris ; les deux aspects pouvaient se rejoindre, mais la méfiance inspirée par la ville provenait aussi d'origines paysannes qui pouvaient constituer un solide repoussoir : "Contre la fascination de Paris, le roman algérien oppose le plus souvent à la ville en général, symbole d'altérité, une légitimité essentiellement terrienne. [...] Souvent les villes d'origine sont évoquées contre Paris depuis Paris même, dans le déchirement de l'exil " (Bonn, 1992 : 211-212). 
intellectuels et étudiants africains, nous le verrons.

Le Paris de I'après-guerre apparaissait ainsi semblable et changeant pour ces migrants éminemment culturels. Rassemblés sous le même vocable, ils répondaient à une infinité de parcours que leurs appréhensions diverses de la capitale laissent déjà entrevoir. Ils étaient vrais, les propos d'Hemingway (2011 : 299) : " II n'y a jamais de fin à Paris et le souvenir qu'en gardent tous ceux qui y ont vécu diffère d'une personne à l'autre ". Ce qui s'appliquait au souvenir valait aussi pour l'expérience.

\section{Le « cosmopolitisme " parisien : passages, métissages et redéfinitions identitaires}

Le discours réflexif offre une base solide, mais I'analyse seule des déclarations ne saurait contenter. Elles doivent être soutenues et confirmées par l'épreuve de la réalité. On a certes postulé le reflet d'une sincérité dans de tels propos, or, comme l'on met ici l'accent sur le lien entre migration et identité chez les intellectuels, les voies de l'existence tangibles importent au moins autant que celles de l'introspection, si précieuses soient-elles. L'identité s'écrit ; elle se vit aussi. L'ancrage réel était-il donc un reflet des diverses perceptions de la Villelumière ? Les nourrissait-il ? La démarche centripète qui convenait à l'examen de l'imaginaire parisien des étrangers ${ }^{16}$ le cède dès lors aux routes escarpées et multiples du réel, un chemin qui voyait s'opérer une mutation de l'identité.

Identité est d'ailleurs un terme générique, d'un emploi souvent abusif, qui recouvre une gamme étendue de référents, allant de l'appartenance à l'identification, voire à la revendication. Les plus grandes précautions méthodologiques s'imposent dès qu'il en est question (Lévi-Strauss, 2010 ; Descombes, 2013). En lien avec l'immigration, ce thème connut dans l'après-guerre une certaine réexploration, même si le terme proprement dit demeurait d'un usage timide (Guedj, 2016). Toujours était-il que l'identité apparaissait comme un élément déterminant de la migration, parfois même comme un fil d'Ariane de l'expérience quotidienne vécue dans le pays d'accueil, a fortiori pour ces intellectuels, particulièrement bien placés pour analyser ce phénomène au "pays qui aime les idées " (Hazareesingh, 2015). Cela s'appliquait surtout aux exilés volontaires, désireux, comme les Américains de la "Génération perdue ", de fuir le conformisme du Nouveau Monde et de découvrir l'authenticité de I'ancien (Schor, 2013 : 19-21). Cette fonction de passage, entre deux mondes, jouait également à plein, autre exemple, pour certains migrants coloniaux, habités, non sans ambivalence, par un désir d'Occident. Abdelkader Khatibi, jeune étudiant quittant un Maroc à peine décolonisé, rappelait son sentiment, certes intellectualisé et maturé par le recul du temps, lors de son départ en 1959 : " Je partais à Paris sans autre histoire que celle d'un étudiant ombrageux, à la recherche d'une autre image des autres et de moi-même. Je me souviens, de même, de ma vacance dans la séparation de deux espaces, légèrement tremblant, assis dans un avion nocturne ; rêve qui,

16 Avec tout ce qu'il contenait de codes et d'exercices de style attendus. Même s'il n'était que touriste, Steinbeck écrivait, ce qui peut s'appliquer aux migrants qui maniaient la plume : "Pour ne pas être ridicule, je dois, lorsque j'écris sur Paris, ne pas chercher à faire du neuf ou de l'original. Aucune ville au monde n'a été mieux aimée ni plus fêtée " (Steinbeck, $1956: 65$ ). 
depuis ma prime enfance, vieillissait dans la narration. Ce vol, rencontrer l'Occident dans le voyage de l'identité et de la différence sauvage " (Khatibi, $2007: 73$ ). Paris, ce concentré d'Occident en même temps si universel, avait le pouvoir de transformer. Dans son roman Un Nègre à Paris, I'Ivoirien Bernard Dadié rapporte I'acuité des états d'âme de son héros: "La bonne nouvelle, mon ami ! la bonne nouvelle ! J'ai un billet pour Paris, oui, Paris ! Paris dont nous avons toujours tant parlé, rêvé. J'y vais dans quelques jours. Je vais voir Paris, moi aussi, avec mes yeux. Désormais, je serai un peu comme tout le monde, je porterai une auréole, un parfum, l'auréole et le parfum de Paris " (Dadié, $1959: 7)$. Une fois " comme tout le monde " - l'impression de se défaire d'un fardeau ? - s'ensuivait un rejet des origines. Le héros le signifiait à un compatriote resté au pays : "N'ai-je pas vécu dans la lumière de Paris ? Je me sens un autre homme et j'oublie jusqu'au souvenir de mes difficultés quotidiennes avec lesquelles tu te bats. Tu es pour moi devenu un attardé, pourri de complexes, de coutumes et de liens imaginaires. Je doute que tes plaintes soient fondées. Paris donne à chacun d'autres sens, une autre mentalité. II vous transforme à votre insu " (Dadié, 1959 : 152). De cet extrait, conçu sans doute pour faire réagir contre les risques de la dépersonnalisation et de la trahison des origines, ressort un schéma d'interprétation proposé par Xavier Garnier. Trois moments se succédaient : " une phase d'enthousiaste émerveillement esthétique ; une phase d'individuation au cours de laquelle le personnage se détache de l'imaginaire colonial de sociétés africaines holistes, où le communautaire est censé primer sur l'individu ; une phase de fraternisation où l'exilé établit des liens avec d'autres exilés, dans une association fraternelle "(Garnier, 2012 : 110). Il régnait donc à Paris un " cosmopolitisme bienfaisant $"{ }^{17}$, mais on trouverait de nombreux témoignages venant contrebalancer celui de Bernard Dadié, qui montreraient au contraire une rancœur née de la " dette de sang " (Dewitte, $1985: 54$ ) non honorée par la métropole après la Première Guerre mondiale, scène que les lendemains de la seconde virent se rejouer. La capitale était donc une " ville mythique pour tous les colonisés [...], à la fois adorée et abhorrée, fascinante et décevante, admirée et conspuée "(Dewitte, $1994: 320)$. Si les migrants coloniaux offraient un cas extrême des rapports tourmentés entre migration et identité, d'autres exilés, volontaires ou forcés, nourrissaient de semblables contradictions. Paris entraînait un changement, une forme de métissage née de la rencontre, mais, par le symbole de l'exil qu'elle représentait - et l'image politique qu'elle renvoyait à certains - elle rendait l'origine omniprésente.

Car la portée du cosmopolitisme, terme largement galvaudé, ne doit pas être exagérée. Pas plus qu'il ne faut accorder une importance démesurée aux ressorts sentimentaux que décrivent les prises de position qui précèdent, empreintes d'une exaltation, dans un sens ou l'autre, qu'une analyse plus froide peut tempérer. Paris a sans doute fait naître une " culture de l'émoi " caractéristique d'un " choc ", semblable à celui qui frappait les visiteurs de la Rome du XVIIle siècle (Bertrand, 2008 : 249-250). Il convient cependant de cesser là les seules références au lien charnel avec la ville. Le processus d'insertion dans des réseaux - littéraires et immigrés -, d'intégration et, vocable qui sied particulièrement ici, d'acculturation, dépendait, dans les faits, de relations nouées

17 Cité par Dewitte (2008: 457) selon les propos recueillis par Ndiaye Jean-Pierre (1962) Enquête sur les étudiants noirs en France, Paris, Réalités africaines, 315 p. 
et entretenues avec des êtres faits de chair, non avec de simples allégories. Vérité d'évidence rappelée par James Baldwin, qui opposait les fantasmes du lointain et les contingences de la réalité, moins chatoyantes : "Pour nous, notre existence et notre travail dépendaient de cette connexion avec l'autre " (Baldwin, $2015: 75)^{18}$. Constat d'échec désabusé. Pourtant, les trajectoires dévoilaient un faisceau de rencontres, de compagnonnages, d'amitiés personnelles doublées de collaborations professionnelles. La force de cette observation varie en fonction de plusieurs paramètres combinés : la date d'arrivée à Paris, l'origine et, partant, les causes ou objectifs de la migration, le degré de notoriété, ainsi que le statut social, déterminant des hiérarchies dans ce monde cosmopolite. Que Paris constituât un creuset artistique et littéraire gommant ou valorisant la qualité d'étranger, cette assertion se passe de justifications. Bien plus, la place des intellectuels étrangers - légaux ou d'origine - paraissait unique sur la scène publique. Ce qui se retrouvait au sein des principales familles de pensée, marquées par de puissantes lignes de clivage qui redéfinirent le monde intellectuel parisien à l'apogée de la Guerre froide (Sirinelli, 1994). Certes, parmi les étrangers, nombre des victimes du stalinisme, s'étant réfugiées en France, nourrissaient les rangs libéraux et antitotalitaires. Tony Judt écrit ainsi : " De fait, après la guerre, l'intelligentsia française libérale était exotique en un sens très précis. Dans ces années-là, les étrangers avaient une importance disproportionnée parmi les collaborateurs des revues ou des publications libérales comme Preuves ou Liberté de l'esprit. Aron et Claude Mauriac y côtoyaient en effet Denis de Rougemont, Ignazio Silone, Nicola Chiaromonte, Manès Sperber, Czeslaw Milosz, Mircea Eliade, Stephen Spender, Karl Jaspers, Bertrand Russell, Sidney Hook, Arthur Koestler, Émile Michel Cioran, Konstanty Jelenski et bien d'autres " (Judt, 1992 : 288). Et d'ajouter : "Paris était encore l'aimant naturel des exilés de toutes sortes de l'Amérique maccarthyste, de I'Europe centrale communiste et des dictatures hispaniques " (ibid. $)^{19}$.

Très fastidieux se révélerait un inventaire à la Prévert des relations intellectuelles, littéraires et, donc, souvent politiques, qui unissaient Français et allogènes, lesquelles se superposaient à celles entretenues au sein d'une même nationalité, non moins simples à délimiter et sur lesquelles il faudra revenir. Piochons, pêle-mêle, quelques exemples, plus ou moins célèbres, mais tous révélateurs à leur manière. Le Belge Henry Bauchau, qui séjourna à Paris de 1945 à 1951, attendit cette date, lui qui était né en 1913, pour se détourner des activités

18 Cité et traduit par Baldwin (2015: 75) et extrait de Baldwin James (1961) The New Lost Generation, Esquire, juillet, p. 113.

19 Les lignes de fracture politiques apparaissaient cruciales. Pour autant, les intellectuels étrangers situés dans l'orbite du camp libéral souffraient d'un double décentrement, national et politique. Lisons encore Judt (1992: 288) : "Beaucoup étaient d'anciens communistes (que d'autres allaient rejoindre dans le courant des années 1950), grâce à quoi ils étaient particulièrement bien renseignés et clairvoyants quant au marxisme et à ses conséquences politiques; mais cela ne suffisait pas à leur assurer un public, au contraire. Dans I'atmosphère de l'époque (dans tout I'Occident, mais surtout à Paris), leurs points de vue et leurs arguments étaient souillés par leurs origines, leur expérience et leurs affiliations politiques présentes. [...] Plus que leur anticommunisme, c'était le cosmopolitisme même de leurs origines et de leurs centres d'intérêt (outre qu'ils se faisaient souvent les avocats de l'idée d'une Europe fédérale) qui rendait ces libéraux particulièrement étrangers ". Si l'on suit cette dernière hypothèse, peu reprise parmi les historiens qui préfèrent parler de creuset intellectuel, on se rend compte que l'origine des hommes de l'esprit avait des conséquences différentes en fonction des cercles dans lesquels ils se mouvaient. 
jugées sérieuses dans le milieu bourgeois d'où il était issu, et s'adonner à ses passions littéraires; il se rapprocha d'Albert Camus, André Gide, Jean Paulhan, mais aussi de Jean Amrouche. À son retour à Paris après la guerre, I'Égyptien Albert Cossery fréquenta là encore Albert Camus, Jean Genet et Robert Nimier. Quant à Bruno Durocher, poète d'origine polonaise installé en France en 1945 à sa libération des camps de concentration, il créa, en 1949, la revue Caractères, suivie d'une maison d'édition du même nom, réalisations qui furent très favorablement accueillies, de même que son premier recueil Chemin de couleur (1949), par le milieu littéraire parisien, plus particulièrement Paul Éluard, Blaise Cendrars, Pierre Jean Jouve, Pierre Reverdy, René Char ou Jules Supervielle ${ }^{20}$. Les accointances politiques, surtout à cette époque, tenaient leur part : c'était le communisme qui rassemblait par exemple Richard Wright et Daniel Guérin, même si les engagements d'intellectuels au sein de mouvements politiques proprement dits, au-delà des cercles de pensée et de pression, apparaissaient relativement rares. Jiri Veltrusky, plus connu sous son pseudonyme de Paul Barton (Barton et Rousset, 1959), après avoir quitté la Tchécoslovaquie en février 1948, devint pourtant une figure du syndicat Force ouvrière. II jouissait du soutien d'Irving Brown, représentant pour I'Europe de l'American Federation of Labor, en première ligne de la lutte contre le stalinisme (Grémion, 2011 : 14). Ces voix de l'intelligentsia étrangère exilée comptaient ainsi bien au-delà des cénacles étrangers et acquéraient une centralité notable sur la scène française des idées, qui confirme les propos de Tony Judt. Le cas de François Fejtö, certes installé dans l'Hexagone avant la guerre, ne manque pas d'éclat, tant il reflétait une position d'influence multiforme : conseiller culturel de l'ambassade de Hongrie à Paris, il avait cru possible de collaborer avec les communistes à la reconstruction de son pays, mais finit par rompre avec le régime et se voulut un éveilleur d'opinion au moment du procès Rajk, en 1949. Figure fondatrice de L'Observateur, journaliste à l'Agence France-Presse, il s'agissait de " l'intellectuel de référence principal " (Grémion, $1995: 252$ ) d'un courant d'abord conciliateur, avant de devenir " I'homme de la situation à I'automne 1956 " (ibid. : 253), au sein de diverses revues comme Les Lettres Nouvelles ou Le Figaro littéraire, en plus de L'Observateur ou du Monde. De ces diverses trajectoires, ressort I'idée que l'origine ne constituait plus qu'un référent d'identité et d'action parmi d'autres.

Les différents espaces de sociabilité où évoluaient ces intellectuels étrangers semblent appuyer cette hypothèse. Les journaux ou revues, on l'a souligné, constituaient des lieux de rencontre privilégiés. Certaines, françaises et étrangères, allèrent jusqu'à entretenir une sorte de jumelage : toujours dans le contexte omniprésent de la lutte des blocs, Preuves, émanation, en 1951, du bulletin de liaison du Congrès pour la liberté de la culture, dont le secrétariat international venait de prendre quartiers à Paris, se rapprocha très étroitement de Kultura, organe anticommuniste polonais créé en 1947 par Jerzy Giedroyc. Pierre Grémion note qu' " aucune revue française publiée dans I'après-guerre n'eut de relation aussi étroite avec une revue d'émigration. Sans aller jusqu'à parler de revues sœurs, on peut assimiler Preuves et Kultura à des cousines germaines très proches "(Grémion, $2011: 41)$. Les comités de rédaction de

20 Ces éléments biographiques proviennent de Mathis-Moser et Mertz-Baumgartner (2012) 
certains vecteurs d'expression et de pensée faisaient voisiner une constellation $d^{\prime}$ individus d'horizons et tendances divers, ce qui tenait autant au hasard des rencontres et des liens qu'à une stratégie élaborée, comme c'était le cas de Présence Africaine, née en 1947. À son comité de patronage signaient en effet les chantres de la littérature pure, tels André Gide, des universitaires spécialistes de l'Afrique, comme Paul Rivet et Théodore Monod; les chrétiens de la tendance $d^{\prime} E s p r i t$ se trouvaient représentés par Emmanuel Mounier, le père Jean-Augustin Maydieu ou Léopold Sédar Senghor ; la mouvance animée par Les Temps modernes n'était pas en reste, avec Albert Camus, Jean-Paul Sartre, Michel Leiris et Aimé Césaire. Enfin, les équilibres régionaux, à l'intérieur de la sphère de la " négritude ", paraissaient respectés : I'Américain Richard Wright et Paul Hazoumé, originaire du Dahomey, travaillaient de concert avec des AfroAntillais comme Aimé Césaire et Senghor (Malela, 2008 : 220 ; Mouralis, 1992), déjà cité, bien implantés dans le paysage culturel et politique français, puisqu'ils siégeaient à l'Assemblée nationale et multipliaient les cercles d'appartenance (Chathuant, 2009). Buata Malela, qui rappelle le soubassement politique de ces liens, puisque "Paris est un centre privilégié où tout le pouvoir symbolique et politique de l'empire français se trouve concentré ", évoque également la force du lien interpersonnel et des intérêts qui le régissaient. Ainsi, le directeur de la revue, Alioune Diop, fait " bénéficier Présence Africaine de la légitimité de ses agents consacrés, puisque la plupart des membres du comité de patronage occupent des positions importantes dans le champ intellectuel parisien " (Malela, $2008: 219,220)$. La présence de membres du Comité national des écrivains (CNE), d'universitaires et écrivains de renom, prêtait du prestige à une revue qui, en retour, soutenait leur engagement - on pense ici à Sartre.

D'autres lieux de rencontre, comme les maisons d'édition, dans le plein éclat des littératures étrangères après 1945, comptèrent également et se firent les vecteurs d'un bouillonnement intellectuel (Mollier, 1994). Qui dit rencontre, ne signifie pas nécessairement relation. Les simples croisements, les échanges d'amabilité étaient souvent le seul visage de ces liens où les microcosmes se frôlaient plus qu'ils ne se fondaient. Aussi Pascal Ory invite-t-il à distinguer, sous la bannière trop générale de la sociabilité, les " colonies ", fondées sur un regroupement géographique, et les "bandes ", liées par un ciment principalement professionnel. Certes, "les deux axes se croisent fréquemment, comme on le devine, l'immigrant culturel ayant une compréhensible tendance à s'agréger à un groupe qui réunira à ses yeux le maximum de signes de "reconnaissance", dans les deux sens du mot " (Ory, $1994: 367)$.

C'était donc que deux niveaux de référence et d'existence se superposaient. Culturellement, le mot de Brancusi, selon lequel "En art, il n'y a pas d'étranger ", conservait toute sa force. Sur le plan identitaire, qui entretenait, dans le contexte de l'exil, une tension étroite avec l'aspect précédent, le creuset intellectuel parisien se heurtait à plus d'une résistance. Voulue ou inconsciente. 


\section{Derrière le cosmopolitisme, l'entre-soi ? Permanences identitaires et contingences migratoires}

À l'orée des années 1950, un important ouvrage, Enracinement des immigrés, s'attacha à distinguer divers " types " d'immigrés. La catégorisation, caractéristique de l'époque, fonctionnait ici à plein, même si la bonne foi des auteurs ne fait pas doute. Au détour d'une page se profilait la question des intellectuels immigrés. Nul besoin de longs développements, car l'affaire semblait entendue : ils n'avaient rien d'immigrés traditionnels. Tant leur vocation que leur talent ou leur place dans la société les retranchaient du monde des immigrés au sens courant du terme : "L'immigration en France se trouve, soit naturellement, soit à la suite d'un déclassement social, affecter surtout des gens simples. Quant à la réussite brillante dans notre pays de certains intellectuels étrangers ou réfugiés, elle ne signifie nullement la réussite d'une assimilation collective : Diaghilev, Chagall, Apollinaire ne sont pas les produits réussis d'une immigration, mais des types d'intellectuels internationaux dont la vie se situe à un niveau culturel et social qui ignore les dossiers de naturalisation " (Nourissier et Pillépich, 1951 : 20). Même si l'on réduit la portée, beaucoup trop large ici, du terme d'intellectuels, cette observation, comme l'attestent les illustres exemples choisis, ne valait que pour une poignée d'individus, les plus en vue et installés. Que faire de l'océan des précaires, des déracinés en mal d'inspiration déplorant leur déclassement culturel et social, des écrivains du dimanche que l'exil orienta vers la plume, mais qui conservaient d'autres ancrages ? Certes, il existait à proprement parler des professions intellectuelles (Sapiro, 2006) ; le statut d'écrivain pouvait conférer certains droits concrets ${ }^{21}$. De rares élus pouvaient y prétendre. Le quotidien de beaucoup, n'était peut-être l'activité principale, correspondait à celui de la grande masse des migrants ${ }^{22}$. Ils appartenaient, par la culture, à une migration d'élite, même si les destins se redessinaient à l'arrivée en France, mais cela ne s'accompagnait en aucun cas nécessairement d'une semblable position sur le plan social ${ }^{23}$. Même si elle demeure en marge de notre propos, la situation de certains étudiants apparaissait particulièrement préoccupante, au point qu'ils durent compter sur les secours de la solidarité et de la bienfaisance ${ }^{24}$. Ceux qui, plus tard devenus des intellectuels reconnus, avaient eu à souffrir d'une telle précarité, la relièrent parfois intimement à leur condition. Tel James Baldwin, lorsqu'il évoquait les problèmes de logement : "Face à cette flambée des loyers, nous, qui nous pensions installés à vie dans le Quartier Latin, dûmes chercher un peu partout dans Paris. Cela se révéla cependant, au moins pour certains d'entre nous, très sain et bénéfique. Nous étions à Paris, après tout, parce que nous refusions les formules et la sécurité et souhaitions connaître le frisson de

21 Archives Nationales, $\mathrm{F}^{1 \mathrm{a}} 3345$, bilan dressé par la Direction des étrangers et des passeports au ministre de I'Intérieur (Adrien Tixier), 7 février 1945.

22 Souvent pour des raisons politiques, beaucoup eurent du mal à obtenir leurs papiers : Richard Wright le dut au soutien de Gertrude Stein ; Pablo Neruda avait gagné la France, en mars 1949, avec le passeport de Miguel Angel Asturias et put compter sur le soutien de Picasso et Supervielle pour régulariser sa situation.

23 Sur la question de la migration des élites, bien plus large que celle des seuls intellectuels, nous renvoyons aux travaux de Green (2008 et 2009).

24 Les étudiants d'outre-mer trouvent difficilement à se loger, Le Monde, 10 octobre 1953 ; Alexandre Biyidi (pseudonyme de Mongo Béti) (1953) Problèmes de l'étudiant noir, Présence Africaine, 14. 
l'expérience et de ses imprévus ${ }^{25}$. Et de donner l'exemple d'un jeune candidat au succès, qui dut se replier sur ce qu'il pensait être les réseaux américains, pour percer : "Un garçon, par exemple, s'était embarqué dans une carrière qui, je crois, est toujours la sienne, laquelle consistait à écrire de laborieuses pièces de théâtre extrêmement littéraires en anglais pour ensuite les traduire laborieusement - en français et en espagnol, avant d'en lire le résultat à une clique d'amis, déjà de moins en moins nombreux, pour ensuite remiser ses manuscrits dans une malle fermée à clef. Des magazines poussaient comme des champignons et disparaissaient aussi vite qu'une nappe de brouillard. Certains peintres et poètes, médiocres et paresseux, souffrant du manque d'intérêt des Français pour leurs efforts, s'employèrent à gagner de manière outrageusement ostentatoire - et efficace - l'attention de figures littéraires américaines en visite, dont les capacités de travail ne faisaient, elles, aucun doute ${ }^{26}$. La gloire tardait donc à se montrer pour le plus grand nombre, qui ne pouvait vivre de sa plume. Jacques Sternberg, né à Anvers, s'installa à Paris en 1945, mais connut une vie si misérable qu'il regagna la Belgique, avant de retenter l'expérience, en 1951, où il exerça les métiers d'emballeur, de dactylo, de représentant de commerce, tout en publiant.

Autant d'obstacles contingents qui entravaient la création. L'exil privait en outre d'un public, d'une inspiration que le climat artistique ne suffisait pas toujours à combler. C'est sans doute à Henri Troyat - arrivé en France en 1917, prix Goncourt en 1938 avant d'entrer à l'Académie française en 1959 - que I'on doit, parmi une littérature abondante, les pages les plus pénétrantes : "Comme pour m'inciter à la modestie au milieu de mon succès, je fis connaissance, vers cette époque, avec plusieurs écrivains russes émigrés, au moment de la révolution bolchevique : Merejkovsky, Zénaïde Hippius, Bounine, Remizov, Chmelov. Tous me dirent qu'ils se réjouissaient de ma distinction, mais je percevais, à travers leurs propos, la profonde tristesse des grands créateurs privés de leur audience. Ils illustraient à mes yeux, le problème tragique des intellectuels exilés. [...] En s'expatriant, ils avaient perdu le public de leurs débuts sans en acquérir un nouveau. Les éditeurs hésitaient à publier des traductions de leurs œuvres, la presse ne les soutenait pas, seul un petit cercle d'émigrés russes les encourageait à poursuivre. Tout se passait comme si, arrachés à la foule de leurs compatriotes, qui faisaient leur gloire en Russie, ils avaient brusquement perdu leur raison d'être. Comme si, aux regards mêmes de ceux qui les avaient accueillis si généreusement, ils représentaient des citoyens déracinés et, par conséquent, des valeurs sujettes à caution. Comme si les écrivains n'avaient de qualité que s'ils exerçaient leur métier dans les limites géographiques de leur pays natal. Partis de Russie, ils n'étaient arrivés nulle part, ils continuaient à vivre dans une zone intermédiaire abstraite. C'était l'enfer glacé des apatrides" (Troyat, 1976 : 81-82). Se complaire dans I'entre-deux, dans I'extranéité subie, constituait une forme de revendication identitaire. Jorge Semprún tenait à ses origines même s'il tenait à entretenir une forme de doute dans la sphère publique : " Je n'ai pas envie d'[...]expliquer pourquoi je parle tout à fait comme eux $[\ldots]$, sans accent, c'est-à-dire avec un accent bien de chez eux. C'est le plus sûr moyen de préserver ma qualité d'étranger à laquelle je tiens par-dessus

25 Baldwin James (1961) The New Lost Generation, Esquire, juillet, p. 73.

26 Ibid., pp. 76-77. 
tout " (Semprún, 2012 : 148). Le Juif roumain Ghérasim Luca, arrivé en France en 1952, garda quant à lui volontairement son statut d'apatride (Mathis-Moser et Mertz-Baumgartner, 2012 : 541). Un soutien concret fut apporté avec la naissance du Comité d'aide exceptionnelle aux intellectuels réfugiés (CAEIR), en 1951, qui s'inscrivait dans le sillage d'organismes tels que I'Entraide universitaire et reconnaissait en un sens les deux pôles de l'intellectuel immigré, en l'occurrence réfugié, son origine et son activité (Kaplan, 1996).

Et puis, il y avait les écrivains nés de l'exil, ceux que leur trajectoire et les malheurs de leur condition conduisirent vers la création. Le Roumain Edgar Reichmann, arrivé en France en 1957, ne publia par exemple son premier roman, Le Dénonciateur, qu'en 1962. Cioran dressait, dans un chapitre de La Tentation d'exister intitulé "Avantages de l'exil ", un portrait acerbe de ces écrivains que I'exil avait façonnés: "C'est à tort que l'on se fait de l'exilé l'image de quelqu'un qui abdique, se retire et $\mathrm{s}^{\prime}$ efface, résigné à ses misères, à sa condition de déchet. À l'observer, on découvre en lui un ambitieux, un déçu agressif, un aigri doublé d'un conquérant. [...] Tel écrit un roman qui, du jour au lendemain, le rend célèbre. II y raconte ses souffrances. Ses compatriotes, à l'étranger, le jalousent : eux aussi ont souffert, peut-être davantage. Et l'apatride devient - ou aspire à devenir - romancier. II en résulte une accumulation de désarrois, une inflation d'horreurs, de frissons qui datent. On ne peut indéfiniment renouveler l'enfer, dont la caractéristique même est la monotonie, ni non plus le visage de l'exil " (Cioran, 2011 : 302). D'où il résultait une forme de recherche de l'entre-soi : " Si peu nombreux que soient les émigrés, ils se constituent en groupes, non point pour défendre leurs intérêts, mais pour se cotiser, se saigner, afin de publier leurs regrets, leurs cris, leurs appels sans écho "(Cioran, 2011 : 303). Cette dernière observation laisse à penser que l'exil - même parisien - maintenait les frontières nationales, les redessinait ou même les faisait apparaître clairement dans le contexte de l'éloignement. La condition d'intellectuel et celle d'immigré se conjuguaient pour maintenir des rassemblements sur des bases nationales, culturelles, ethniques, etc. Identitaires en d'autres termes.

Revendiquer, plus ou moins ouvertement, son origine pourrait de prime abord sembler freiner le mouvement d'intégration au pays d'accueil et plus particulièrement au milieu parisien de l'esprit. Or, malgré les phénomènes de rencontre et de métissages décrits, c'était bien en tant qu'étrangers qu'ils occupaient leur place. Reprenant le fameux " dilemme de Ramuz "27 (Casanova, 1999: 246), on peut, comme le fait Buata Malela pour les Afro-Antillais, I'appliquer à une variété de cas. Alioune Diop, Ousmane Socé et Léopold Sédar Senghor répondaient à cette tension : " soit ils affirment leur différence (écrivain nègre) et, dès lors, se condamnent à la marginalisation littéraire, soit ils s'assimilent au centre littéraire en reniant leur différence - comme l'a tenté Maran -, et dans

27 Formulé par l'écrivain suisse dès 1935 en ces termes : " C'est le dilemme qui s'est posé pour moi, quand j'avais vingt ans, et qui se pose pour tous ceux qui sont dans le même cas que moi, qu'ils soient nombreux ou pas nombreux : les extérieurs, les excentriques, ceux qui sont nés hors d'une frontière; ceux qui, tout en étant liés à une culture par la langue, sont en quelque sorte exilés d'elle par la religion ou par leur appartenance politique [...]. Le problème se pose tôt ou tard : ou bien il faut faire carrière et $\mathrm{d}^{\prime}$ abord se plier à un ensemble de règles qui ne sont pas simplement esthétiques ou littéraires, mais encore sociales, politiques ou même mondaines; ou bien rompre délibérément avec elles, non seulement en laissant voir, mais en exagérant ses propres différences : quitte à les faire admettre plus tard si on peut "(Questions, cité par Casanova, 1999). 
ce cas, augmentent leurs chances de parvenir à une plus grande visibilité littéraire. [...] La difficulté pour eux consiste à rejeter I'assimilationnisme politique et en même temps à éviter la marginalisation littéraire en affirmant subtilement leur différence " (Malela, 2008 : 140). Une entreprise comme Présence Africaine dépassait déjà largement cette tension et contribuait à cerner un groupe, celui des Noirs - indépendamment des origines nationales -, d'une frontière visible quoique non étanche (Diop, 1949). En 1956, se tint à la Sorbonne le premier Congrès international des écrivains et artistes noirs. On trouverait facilement un groupe voisin, celui des "Maghrébins ", qui se réunirent en 1955, lors d'un débat au collège philosophique, représentés par le Juif tunisien Albert Memmi, l'Algérien Kateb Yacine et le Marocain Driss Chraïbi (Arnaud, 1992 : 201). Paris voyait la naissance d'une " littérature nord-africaine ${ }^{28}$. Dans le cas des intellectuels latino-américains, la prise de conscience de l'unité de leur culture et de leur continent - dont les centres échangeaient plus avec les capitales européennes qu'entre eux - devait passer par l'éloignement à Paris (Weiss, $2003: 2$ ).

Dans ces conditions, parler de repli identitaire paraîtrait exagéré. En revanche, selon la nature du lien qui unissait certains microcosmes, il arrivait que les contacts avec l'extérieur fussent réduits. Si un Richard Wright ou un Ollie Harrington manifestèrent un intérêt pour les revendications indépendantistes algériennes, bien souvent, les préoccupations concernaient les origines directes des intellectuels étrangers. L'audience pouvait ainsi apparaître élevée parmi les compatriotes, mais faible dans le milieu politique et littéraire parisien. Deux critiques littéraires, Monica Lovinescu et Virgil lerunca, arrivés en France à la fin des années 1940, s'engagèrent contre le communisme en Roumanie et disposèrent de solides relais ; " ce rôle et cette mission les ont orientés presque exclusivement vers l'espace politique et culturel roumain, ce qui les a privés d'une insertion plus active dans le milieu parisien, où ils étaient connus surtout parmi les autres exilés provenant de l'Europe de I'Est et parmi les chercheurs français du totalitarisme " (Carneci, $2011: 53$ ). Les relations internes ne s'en trouvaient pas pour autant facilitées, chaque groupe traçant ses propres frontières et clivages intérieurs. En fonction de la date d'arrivée, de la position sociale et de la place réservée aux intellectuels au sein de cellules nationales. Milosz l'a remarquablement évoqué : "II existe un conflit entre les politiciens d'émigration et les intellectuels. C'est quelque chose de plus que le conflit classique entre émigrations, vieille et nouvelle. On peut y trouver les traces du conflit entre deux groupes sociaux ". Et d'opposer l'intelligentsia cultivée, composée d'individus éclairés qui ne faisaient pas de l'esprit leur matière d'activité principale, aux " intellectuels proprement dits [qui] sont ce que l'on appelle l'intelligentsia créatrice " (Milosz, $1951:$ 7). L'exil ne rassemblait pas, mais, imprimant de son sceau toutes les expériences, les diffractait en une diversité de parcours.

"Le lendemain même de son arrivée à Paris, Khaled savait qu'un roman commençait dont l'exil serait plus l'auteur que le cadre ". Les pensées du héros peint par Malek Haddad (Haddad, 1961 : 17) pourraient synthétiser à elles seules le propos que l'on a tenté de développer. L'intellectuel en exil était aussi un intellectuel de l'exil, ce dernier élément agissant d'une manière déterminante et constante. Cette catégorie générique n'en apparaissait pas moins travaillée

28 Voir Études, février 1956 ; Documents nord-africains, 240, 19 septembre 1956, ainsi que I'étude de Scagnetti, dans le présent dossier. 
par des tensions, des différences profondes, qui inviteraient à se méfier des grandes généralisations. C'est pourquoi on a préféré une analyse en termes de parcours plutôt que de groupes, trop figés pour ne pas être étudiés de manière par trop réductrice. Telle est la difficulté qui consister à pénétrer l'univers des " migrations fines" (Simon, 1995 : 40), par le nombre et la nature fuyante. Entre l'exilé volontaire et le réfugié, les points communs ne manquaient pas, mais l'expérience relevait d'ordres distincts; en revanche, en dépit d'évidentes spécificités, les diverses origines se prêtent parfaitement à la comparaison, qui convainc de plaider pour une approche globale des phénomènes migratoires. Les transferts culturels marquaient tous ces intellectuels qui se retrouvaient à Paris dans l'après-guerre, mais ne se dépouillaient pas de leur identité, qu'ils pouvaient même réexplorer et redécouvrir dans l'éloignement et la quête de sens que la Ville-lumière rendait propice. Retrouver son identité dans le creuset d'une nouvelle appartenance : tel est sans doute le pouvoir conféré par Paris, tout à la fois ville et monde.

\section{Références bibliographiques}

Arnaud Jacqueline (1992) Le Paris des Maghrébins : regroupement et ouverture, in Université de Paris IV Éd., Paris et le phénomène des capitales littéraires. Carrefour ou dialogue des cultures, Paris, Presses de I'Université de Paris Sorbonne, pp. 194-202.

Baldwin James (2015) Retour dans l'œil du cyclone, Paris, Christian Bourgois, $226 \mathrm{p}$.

Baldwin James (2000 [1948]) The Devil Finds Work, New York, Random House, $144 \mathrm{p}$.

Baldwin James (1998 [1956]) La Chambre de Giovanni, Paris, Payot \& Rivages, $237 \mathrm{p}$.

Barton Paul et Rousset David (1959) L'Institution concentrationnaire en Russie, précédé du Sens de notre combat, Paris, Plon, 519 p

Bertrand Gilles (2008) Le Grand Tour revisité. Pour une archéologie du tourișme : le voyage des Français en Italie (milieu XVIle-début XIXe siècle), Rome, École française de Rome, $791 \mathrm{p}$.

Bonn Charles (1992) Paris et la description des villes d'identité par quelques romans algériens, in Université de Paris IV Éd., Paris et le phénomène des capitales littéraires. Carrefour ou dialogue des cultures, Paris, Publications de la Sorbonne, pp. 211-220.

Caillois Roger (1938) Paris, mythe moderne, in Roger Caillois, Le Mythe et I'homme, Paris, Gallimard, pp. 153-175.

Carneci Magda (2011) L'exil littéraire roumain en France : entre résistance politique et nouvel œcuménisme, in Wojciech Falkowski et Antoine Marès Dirs., Intellectuels de l'Est exilés en France, Paris, Institut d'Études Slaves, pp. 49-61.

Casanova Pascale (1999) La République mondiale des Lettres, Paris, Le Seuil, $607 \mathrm{p}$.

Chathuant Dominique (2009) L'émergence d'une élite politique noire dans la France du premier XXe siècle ?, Vingtième Siècle, 101, pp. 133-147. 
Cioran Emil (2011 [1956]) La Tentation d'exister, in Emil Cioran Euvres, Paris, Gallimard, pp. 263-428.

Dadié Bernard (1959) Un Nègre à Paris, Paris, Présence Africaine, 217 p.

Delbart Anne-Rosine (2005) Les Exilés du langage. Un siècle d'écrivains français venus d'ailleurs (1919-2000), Limoges, PULIM, $262 \mathrm{p}$.

Descombes Vincent (2013) Les Embarras de I'identité, Paris, Gallimard, 282 p.

Dewitte Philippe (2008) L'immigration : l'émergence en métropole d'une élite africaine (1946-1961), in Pascal Blanchard, Sandrine Lemaire et Nicolas Bancel Dirs., Culture coloniale en France. De la Révolution française à nos jours, Paris, CNRS Éditions, pp. 451-459.

Dewitte Philippe (1994) Intellectuels et étudiants africains à Paris à la veille des indépendances (1945-1960), in Antoine Marès et Pierre Milza Dirs., Le Paris des étrangers depuis 1945, Paris, Publications de la Sorbonne, pp. 319-342.

Dewitte Philippe (1985) Les Mouvements nègres en France (1919-1939), Paris, L'Harmattan, $416 \mathrm{p}$.

Dib Mohammed (1958) Jours et Nuits de l'exilé, Les Lettres françaises, 24 avril 1958.

Diop Alioune (1949) Malentendus, Présence Africaine, 6, p. 286.

Dollé Marie (2001) L'Imaginaire des langues, Paris, L'Harmattan, 192 p.

Fabre Michel (1985) La Rive noire. De Harlem à la Seine, Paris, Lieu commun, $337 \mathrm{p}$.

Fejtö François (1986) Mémoires de Budapest à Paris, Paris, Calmann-Lévy, 323 p.

Flanner Jannet (1965) Paris Journal, 1944-1965, New York, Athneum, 615 p.

Flores Angel (1992) Spanish American Author: The Twentieth Century, New York, Wilson, $915 \mathrm{p}$.

Garnier Xavier (2012) Intellectuels africains en exil à Paris : un paradoxe colonial, in Xavier Garner et Jean-Philippe Warren Dirs., Écrivains francophones en exil à Paris. Entre cosmopolitisme et marginalité, Paris, Karthala, pp. 109-124.

Genette Gérard (1982) Palimpsestes, Paris, Le Seuil, 573 p.

Glaser Georges C. (1951) Secret et violence, Paris, Corrêa, 420 p.

Gourfinkel Nina (1953) Aux prises avec mon temps, t. II : L'Autre Patrie, Paris, Le Seuil, 349 p.

Green Julien (1998) Paris, in Julien Green, Fuvres complètes, t. II, Paris, Gallimard, $1616 \mathrm{p}$.

Green Julien (1975) Journal. IV : L'œil de I'ouragan (1943-1945), in Julien Green, Fuvres complètes, t. IV, Paris, Gallimard, pp. 699-884.

Green Nancy L. (2009) Expatriation, Expatriates and Expats: The American Transformation of a Concept, American Historical Review, 114 (2), pp. 307-328.

Green Nancy L. (2008) La migration des élites : nouveau concept, anciennes pratiques ?, Cahiers du Centre de Recherches Historiques, 41, pp. 107-116. 
Grémion Pierre (2011) Voix d'exil en marge du progressisme parisien, in Wojciech Falkowski et Antoine Marès Dirs., Intellectuels de l'Est exilés en France, Paris, Institut d'Études Slaves, pp. 33-47.

Grémion Pierre (1995) Intelligence de l'anticommunisme. Le Congrès pour la liberté de la culture à Paris (1950-1975), Paris, Fayard, 645 p.

Guedj Jérémy (2016) Les temps de l'esprit. Intellectuels, questions identitaires et immigration au lendemain de la Seconde Guerre mondiale, in Jean-Paul Pellegrinetti Dirs., La Méditerranée en passion. Mélanges d'histoire contemporaine offerts à Ralph Schor, Paris, Classiques Garnier, pp. 291-313.

Guedj Jérémy (2015a) Identités de l'esprit. Reconfigurations des frontières nationales et nouveaux espaces identitaires parmi les intellectuels étrangers exilés en France (1945-1960), in Maroussia Ahmed, Corinne Alexandre-Garner, Nicholas Serruys, Iulian Toma et Isabelle Keller-Privat Dirs., Migrations/Translations, Nanterre, Presses universitaires de Paris-Ouest, pp. 81-100.

Guedj Jérémy (2015b) Gouverner ou choisir. La IVe République et l'immigration, Thèse de doctorat en histoire contemporaine, sous la direction de Ralph Schor, Université Nice-Sophia Antipolis, 946 p.

Haddad Malek (1961) Le Quai aux fleurs ne répond plus, Paris, Julliard, 194 p.

Hazareesingh Sudhir (2015) Ce pays qui aime les idées. Histoire d'une passion française, Paris, Flammarion, $469 \mathrm{p}$.

Hemingway Ernest (2011 [1964]) Paris est une fête, Paris, Gallimard, 350 p.

Judt Tony (1992) Un passé imparfait. Les intellectuels en France (1944-1956), Paris, Fayard, 404 p.

Kaplan Hélène (1996) Le Comité d'aide exceptionnelle aux intellectuels réfugiés, Matériaux pour l'histoire de notre temps, 44, , pp. 59-62.

Kaspi André et Marès Antoine (1989) Le Paris des étrangers depuis un siècle, Paris, Imprimerie nationale, $406 \mathrm{p}$.

Khatibi Abdelkader (2007 [1971]) La Mémoire tatouée. Autobiographie d'un décolonisé, in Abdelkader Khatibi, Romans et récits, t. I, Paris, Différence.

Lapierre Nicole (2004) Pensons ailleurs, Paris, Stock, 301 p.

Laronde Michel (1999) Stratégie post-coloniale de décentrage littéraire. Apprendre à lire autrement, in Christiane Albert Dir., Francophonie et identités culturelles, Paris, Karthala, pp. 285-300.

Lévi-Strauss Claude (Dir.) (2010 [1977]) L'identité, Paris, PUF, 344 p.

Malaparte Curzio (1967) Journal d'un étranger à Paris, Paris, Denoël, 393 p.

Malela Buata B. (2008) Les Écrivains afro-antillais à Paris (1920-1960). Stratégies et postures identitaires, Paris, Karthala, $465 \mathrm{p}$.

Mammeri Mouloud (1957) Le Sommeil du juste, Paris, Plon, 254 p.

Marès Antoine (1994) Exilés d'Europe centrale de 1945 à 1967, in Antoine Marès et Pierre Milza Dirs., Le Paris des étrangers depuis 1945, Paris, Publications de la Sorbonne, pp. 129-168. 
Marès Antoine et Milza Pierre (1994) Le Paris des étrangers depuis 1945, Paris, Publications de la Sorbonne, $470 \mathrm{p}$.

Mathis-Moser Ursula et Mertz-Baumgartner Birgit (2012) Passages et ancrages en France. Dictionnaire des écrivains migrants de langue française (1981-2011), Paris, Honoré Champion, 965 p.

Milosz Czeslaw (1951) La Grande Tentation : le drame des intellectuels dans les démocraties populaires, Paris, Preuves, $23 \mathrm{p}$.

Mollier Jean-Yves (1994) Paris capitale éditoriale des mondes étrangers, in Antoine Marès et Pierre Milza Dirs., Le Paris des étrangers depuis 1945, Paris, Publications de la Sorbonne, pp. 373-394.

Mouralis Bernard (1992) Présence Africaine: Geography of an "Ideology", in Valentin-Yves Mudimbe Dir., The Surreptitious Speech. Présence Africaine and the politics of otherness, 1947-1987, Chicago, The University of Chicago Press, pp. 3-13.

Ndiaye Pap (2008) La Condition noire. Essai sur une minorité française, Paris, Calmann-Lévy, $435 \mathrm{p}$.

Nourissier François et Pillépich André (1951) Enracinement des immigrés, Paris, Bloud \& Gay, 240 p.

Ory Pascal (1994) Paris, lieu de création et de légitimation internationales, in Antoine Marès et Pierre Milza Dirs., Le Paris des étrangers depuis 1945, Paris, Publications de la Sorbonne, pp. 359-371.

Pinçonnat Crystel (2001) New York, mythe littéraire français, Genève, Droz, 325 p.

Pinçonnat Crystel (2000) Paris and the "New Lost Generation", The French Review, 73 (6), pp. 1065-1075.

Sapiro Gisèle (2006) Les professions intellectuelles entre l'État, l'entrepreneuriat et I'industrie, Le Mouvement social, 214, pp. 3-18.

Sapiro Gisèle, (2004) Le principe de sincérité et l'éthique de responsabilité de I'écrivain, in Évelyne Pinto Dir., L'Écrivain, le savant et la philosophie. La littérature, entre philosophie et sciences sociales, Paris, Publications de la Sorbonne, pp. 183-202.

Schor Ralph (2013) Écrire en exil. Les écrivains étrangers en France (1919-1939), Paris, CNRS Editions, $346 \mathrm{p}$.

Schor Ralph (1989) Le Paris des libertés, in André Kaspi et Antoine Marès Dirs., Le Paris des étrangers depuis un siècle, Paris, Imprimerie nationale, pp. 13-33.

Schor Ralph (1985) L'Opinion française et les étrangers, Paris, Publications de la Sorbonne., $761 \mathrm{p}$

Semprún Jorge (2012 [1963]) Le Grand Voyage, in Jorge Semprún, Le Fer rouge de la mémoire, Paris, Gallimard, pp. 89-232.

Simon Gildas (1995) Géodynamique des migrations internationales dans le monde, Paris, PUF, $429 \mathrm{p}$.

Sirinelli Jean-François (1994) Paris au cœur du débat Est-Ouest, in Antoine Marès et Pierre Milza Dirs., Le Paris des étrangers depuis 1945, Paris, Publications de la Sorbonne, pp. 120-128. 
Slama Alain-Gérard (1998) La crise du bonheur français, Commentaire, 81, pp. 121-124.

Stein Gertrude (1941) Paris-France, Alger, Charlot, 211 p.

Steinbeck John (1956) Un Américain à New York et à Paris, Paris, Julliard, 159 p.

Troyat Henri (1976) Un si long chemin, Paris, Stock, 217 p.

Weiss Jason (2003) The Lights of Home. A Century of Latin American Writers in Paris, New York, Routledge, 278 p. 


\section{Jérémy Guedj}

\section{Les espaces de l'intellectuel en exil : trajectoires et réseaux immigrés dans le Paris d'après-guerre (1945-1960)}

Cet article se propose d'explorer la manière dont l'expérience de l'exil a pu reconfigurer les appartenances des intellectuels étrangers venus en France après 1945. De fait, ils évoluaient entre plusieurs espaces et appartenaient à divers réseaux, nationaux ou plus particulièrement étrangers, qui, tout en renforçant I'hybridité de leur condition, les conduisaient à réinterroger en permanence leur identité, comme le reflétaient leurs écrits.

\section{Exiled Intellectuals Spaces: Immigrant Paths and Networks in Afterwar Paris (1945-1960)}

This paper aims at showing how the experience of exile could reconfigure the sense of belonging shared by foreign intellectuals who came in France after 1945. Indeed, they were living between several spaces and were belonging to different networks, which were of national or more particularly foreign origins. By reinforcing their condition hybridity, this phenomenon led those intellectuals to constantly examine their identity, as reflected in their works.

\section{Los espacios del intelectual en exilio: trayectorias y redes de inmigrantes en el París de posguerra (1945-1960)}

Este artículo propone estudiar la manera con la cual la experiencia del exilio pudo reconfigurar las pertenencias de los intelectuales extranjeros llegados a Francia después de 1945. En efecto, aquellos evolucionaban por varios ámbitos y formaban parte de numerosas redes, nacionales o más especificadamente extranjeras, que fortalecían el hibridismo de su condición a la par que les incitaban a no dejar de cuestionar su identidad, como se desprende de sus escritos. 